\title{
The gray area between operational tolerance and overt rejection in kidney transplantation
}

\author{
Rui F. Silva ${ }^{1}$ (D), Miguel L. Relvas ${ }^{1}$ (iD), Ana Nunes ${ }^{1}$ (iD), José L. Silvano ${ }^{1}$ (iD, Ana Beco ${ }^{1}$ iD, Manuela B. Bustorff ${ }^{1}$ iD, Susana M. Sampaio ${ }^{1}$ iD \\ ${ }^{1}$ Nephrology Department, Centro Hospitalar de São João, Porto, Portugal
}

\section{ABSTRACT}

Operational tolerance in kidney transplantation is characterized by stable serum creatinine $<1.7 \mathrm{mg} / \mathrm{dL}$ and proteinuria $<1 \mathrm{~g} / \mathrm{day}$ in the absence of immunosuppression or immunodeficiency for over one year. However, simultaneous donor specific antibodies are common and serum creatinine is a poor surrogate of early lesions. Consequently, subclinical rejections will meet operational tolerance criteria if serum creatinine remains stable. We report a patient with operational tolerance criteria followed by biopsy-proven chronic active antibody mediated rejection, discussing the intricate challenges of immunosuppression management.

Key words: kidney transplantation, operational tolerance, antibody mediated rejection

C 2021 Portuguese Journal of Nephrology \& Hypertension. Published by Publicações Ciência \& Vida This is an open access article under the CC BY-NC-ND license (http://creativecommons.org/licenses/by-nc-nd/4.0/).

\section{INTRODUCTION}

In kidney transplantation, clinical operational tolerance is a rare condition ( 3 per 10.000 kidney transplant patients ${ }^{1}$ ) characterized by stable serum creatinine $(\mathrm{s} C \mathrm{r})<1.7 \mathrm{mg} / \mathrm{dL}$ and proteinuria $<1 \mathrm{~g} /$ day in the absence of immunosuppression (IS) or immunodeficiency for over one year. It may concur with HLA donor specific antibodies (DSAs), without clinically detectable deleterious immune response against the graft, and may be limited to a few years or persist for decades. Due to the lack of reliable biomarkers of tolerance beyond creatinine and proteinuria, some patients may be inappropriately labeled as operationally tolerant in the presence of subclinical rejection ${ }^{2}$. We report a patient with operational tolerance criteria followed by biopsyproven chronic active antibody mediated rejection, discussing the implications of withholding immunosuppression from supposedly operational tolerant patients with DSAs.

\section{CASE REPORT}

A 53-year old female was admitted for acute graft pyelonephritis. Her past medical history included chronic kidney disease of unknown etiology which led to hemodialysis at the age of 34 . She was transplanted at the age of 42 from a standard criteria donor (3/6 HLA mismatches - 1A 1B 1DR), shortly followed by an acute lb T cell mediated rejection (Banff 2017), successfully treated with thymoglobulin and methylprednisolone, and discharged under maintenance IS with tacrolimus, mycophenolate, and prednisolone (s $\mathrm{Cr} 1,02 \mathrm{mg} / \mathrm{dL}$ ). She was lost to follow-up 4 years after transplant, ceasing all IS (last known $\mathrm{sCr}$ $0.8 \mathrm{mg} / \mathrm{dL}$ ). She resurfaced seven years later, at the age of 53, with lower urinary tract symptoms. Urinalysis was compatible with urinary tract infection, without dipstick proteinuria and a $\mathrm{sCr}$ of $1.01 \mathrm{mg} / \mathrm{dL}$. She was successfully treated for acute graft pyelonephritis and later discharged under her previous IS. She was readmitted six months later for similar complaints, again under no IS, with stable graft function ( $\mathrm{s} C \mathrm{r} 1.04 \mathrm{mg} / \mathrm{dL}$ ), without dipstick proteinuria. The patient was found to have lost her decisional capacity due to global cerebral atrophy secondary to cerebrovascular disease, vitamin B12 and folic acid deficiency. Suspecting of tolerance, the patient was kept without IS, under close surveillance, until further study. Four HLA DSAs were found (mean fluorescence indexes: A34 - 10688, B45 - 5833, DQ7 - 24990, DQ4 -2571), with anti-DQ7 as the sole C1q fixing antibody. Flow cytometry crossmatch using donor cells showed $\mathrm{T}$ and $\mathrm{B}$ cell positivity. Four months after the last admission, a slight deterioration of graft function $(\mathrm{s} C \mathrm{r}$ $1.4 \mathrm{mg} / \mathrm{dL}$ ) led to biopsy, after readily reversible causes of graft dysfunction were excluded. Findings were compatible with chronic active $A B M R$ - Figure 1. Treatment with intravenous immunoglobulin, for a cumulative dose of $2 \mathrm{~g} / \mathrm{kg}$, was started and maintenance IS reintroduced (tacrolimus, mycophenolate, and prednisolone). Numerous infections emerged over the following weeks, leading to interruption of initial plans to add rituximab. The patient has since been kept under maintenance IS, with current $\mathrm{sCr} 1.19 \mathrm{mg} / \mathrm{dL}$ and urinary protein (albumin)creatinine ratio of $635(256) \mathrm{mg} / \mathrm{g}$.

\section{DISCUSSION}

Transplant tolerance is the holy grail of transplantation. To be transplanted off IS, without the imminent risk of rejection, would give the patient the opportunity to overcome transplant major hurdles 


\section{Figure 1}

A. Glomerulitis. B. Peritubular capillary basement membrane multilayering. C. Subendothelial expansion. Biopsy report - 19 glomeruli, 1 with sclerosis. Glomerulitis lesions ( $\mathrm{g} 1$ ) and mesangial matrix expansion (mm1). Interstitial lymphocyte infiltration (i1) and fibrosis (10\% - ci1). Tubulitis (t1) and tubular atrophy (ct1). Peritubular capillaritis (ptc2). Arterial fibroelastosis lesions (cv1). No endothelitis. Immunofluorescence: C4d diffusely positive in the peritubular capillaries. IgM and C1q positivity. Electronic microscopy: focal fusion of podocytes foot processes, matrix expansion and mesangium and glomerular basal membrane sclerosis, subendothelial expansion and peritubular capillary basement membrane multilayerin
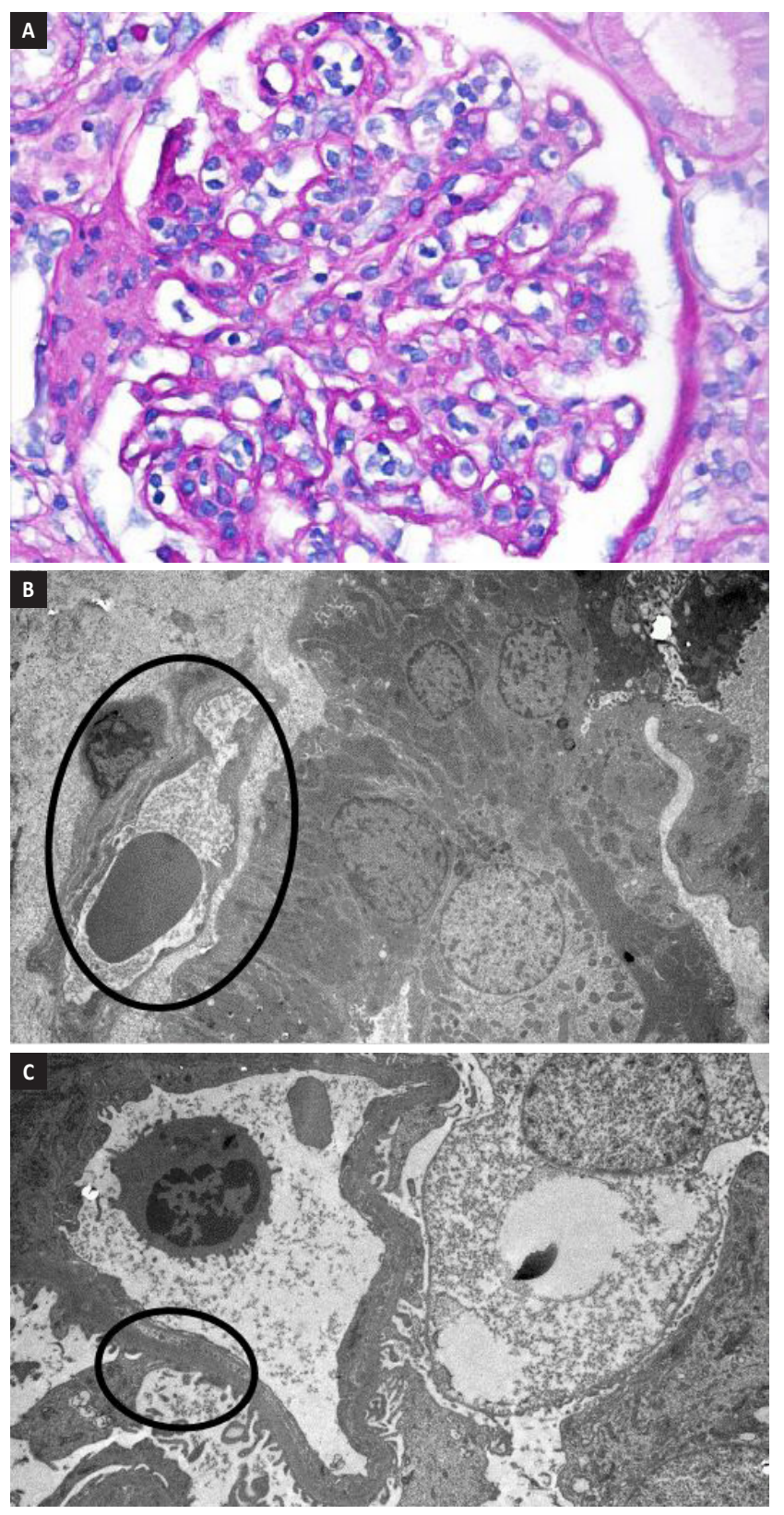

such as infections, malignancies, metabolic complications, nephrotoxicity, and long-term patient and allograft survival. Currently, clinical trials of tolerance induction, through macrochimerism achievement with combined donor kidney and hematopoietic cell transplantation, have moved from preclinical to clinical experimental stages ${ }^{3-5}$. Although revolutionary, wide adoption of these procedures remains encumbered by potentially toxic and life-threatening conditioning regimens, the risk of graft-versus-host disease, and a failure to prevent slow alloimmune responses ${ }^{6-8}$.

Outside clinical trials, however, a few kidney transplant patients, such as the one presented, have been reported to spontaneously maintain a good, prolonged allograft function in the absence of IS or immunodeficiency ${ }^{1}$. These patients are qualified as clinically operationally tolerant based on the presence of a stable $\mathrm{sCr}(<1.7 \mathrm{~g} / \mathrm{dL})$ and proteinuria ( $<1 \mathrm{~g} /$ day) for over one year. In experimental animal models, however, the definition of immune tolerance relies on more strict criteria: 1) preserved graft in the absence of IS, maintaining normal function, histology, and the absence of in vitro donor-specific response; 2) acceptance of a second graft from the same donor; 3 ) rejection of a different donor's graft ${ }^{2}$. Understandably, this is not all possible to demonstrate in a clinical scenario, meaning we cannot assume all operationally tolerant patients to be truly immunologically tolerant. Firstly, because operational tolerance often concurs with the presence of HLA DSAs, suggesting some degree of anti-donor reactivity $^{2}$. Secondly, because serum creatinine is a poor surrogate of early kidney lesions. Since histological lesions may precede renal function deterioration, a proportion of patients with operational tolerance criteria may actually have subclinical rejection. However, misinterpreting chronic active ABMR, such as this patient's, for tolerance could imply an important risk for graft failure ${ }^{9,10}$.

This case makes us consider how to proceed in operationally tolerant patients, clinically well under no IS, but with laboratory findings consistent with $A B M R$. There is no clear answer. On one hand, these patients may preserve graft function for years without IS, even in the presence of HLA antibodies. On the other hand, operational tolerance is a labile state and quick renal function deterioration is a persistent risk, particularly with complement fixing DSAs ${ }^{11}$ and positive crossmatches, as was the case of this patient. IS has turned kidney transplantation into an everyday accessible procedure to end-stage kidney disease patients, able to improve their overall survival and quality of life, compared to dialysis. However, while powerful in avoiding acute rejection, it also condemns circulating donor cells which would have been able to induce immunoregulatory host responses towards the allograft. Possibly because of it, longterm allograft survival is stagnant, overwhelmed with chronic immune injuries and IS toxicities².

In conclusion, we were faced with a rare case of a female patient with complement fixing DSAs and positive crossmatches, fulfilling clinical criteria of operational tolerance, as evidenced by a normal $\mathrm{sCr}$ and proteinuria after seven years off IS. She was admitted twice for urinary tract infection. Operational tolerance does not imply a lack of anti-donor reactivity, as DSAs are often simultaneously present, but the absence of clinically detectable deleterious immune responses against the graft, resembling immunological accommodation in ABO-incompatible kidney transplants, a self-protection mechanism against anti-donor blood-group antibody-mediated damage ${ }^{12}$. Even though the presence of DSAs does not preclude long-lived transplants off IS, it is nonetheless a ringing bell for possible 
subclinical rejection, as creatinine stands as a poor surrogate for early lesions, and IS weaning and non-compliance have been shown to be associated with an increased risk for rejection and graft loss ${ }^{2}$. Understandably, operational tolerance has been called by some authors as a misleading term ${ }^{13}$, as current creatinine/proteinuria binary criteria may encompass three groups of patients: 1) without anti-donor reactivity (DSAs), 2) with DSAs but without rejection and 3) with DSAs and subclinical rejection. While the first two may be indeed considered operationally tolerant, the third is not, which means we can only suppose of tolerance in the absence of a kidney biopsy. If this woman was ever operationally tolerant, she was no longer at the time of biopsy, which led us to definitively restart IS. One hypothesis is that she was indeed operationally tolerant, but urinary tract infection triggered a deleterious immune reaction, as untreated urinary tract infection stands as a risk factor for rejection ${ }^{14}$. There is a paucity of reliable immune tolerance biomarkers beyond creatinine and/or proteinuria guiding clinical decisions on IS withdrawal in this gray area between operational tolerance and overt rejection. Possible new candidates are phenotypical studies of circulating $T$ and $B$ cells and blood transcriptional gene signature ${ }^{2}$. Furthermore, we believe kidney surveillance biopsies may be useful to exclude subclinical pathology associated with shortened graft survival in supposedly operational tolerant patients.

Disclosure of potential conflicts of interest: none declared.

\section{References}

1. Massart A, Pallier A, Pascual J, et al. The DESCARTES-Nantes survey of kidney transplant recipients displaying clinical operational tolerance identifies 35 new tolerant patients and 34 almost tolerant patients. Nephrology, dialysis, transplantation: official publication of the European Dialysis and Transplant Association - European Renal Association. 2016; 31: 1002-1013.

2. Massart A, Ghisdal L, Abramowicz M and Abramowicz D. Operational tolerance in kidney transplantation and associated biomarkers. Clinical and experimental immunology. 2017; 189: 138-157.

3. Kawai T, Cosimi AB, Spitzer TR, et al. HLA-mismatched renal transplantation without maintenance immunosuppression. The New England journal of medicine. 2008; 358: 353-361.

4. Leventhal J, Abecassis M, Miller J, et al. Chimerism and tolerance without GVHD or engraftment syndrome in HLA-mismatched combined kidney and hematopoietic stem cell transplantation. Science translational medicine. 2012; 4: 124ra28.

5. Scandling JD, Busque $S$, Dejbakhsh-Jones $S$, et al. Tolerance and chimerism after renal and hematopoietic-cell transplantation. The New England journal of medicine. 2008; 358: 362-368.
6. Kawai T, Sachs DH, Sprangers B, et al. Long-term results in recipients of combined HLA-mismatched kidney and bone marrow transplantation without maintenance immunosuppression. American ournal of transplantation: official journal of the American Society of Transplantation and the American Society of Transplant Surgeons. 2014; 14: 1599-1611.

7. Leventhal JR, Elliott MJ, Yolcu ES, et al. Immune reconstitution/immunocompetence in recipients of kidney plus hematopoietic stem/facilitating cell transplants. Transplantation. 2015; 99: 288-298.

8. Scandling JD, Busque S, Shizuru JA, et al. Chimerism, graft survival, and withdrawal of immunosuppressive drugs in HLA matched and mismatched patients after living donor kidney and hematopoietic cell transplantation. American journal of transplantation: official journal of the American Society of Transplantation and the American Society of Transplant Surgeons. 2015; 15: 695-704.

9. Redfield RR, Ellis TM, Zhong W, et al. Current outcomes of chronic active antibody mediated rejection - A large single center retrospective review using the updated BANFF 2013 criteria. Human immunology. 2016; 77: 346-352.

10. Moreso $\mathrm{F}$, Ibernon $\mathrm{M}$, Gomà $\mathrm{M}$, et al. Subclinical rejection associated with chronic allograft nephropathy in protocol biopsies as a risk factor for late graft loss. American journal of transplantation: official journal of the American Society of Transplantation and the American Society of Transplant Surgeons. 2006; 6: 747-752.

11. Yabu JM, Higgins JP, Chen G, Sequeira F, Busque S and Tyan DB. C1q-fixing human leukocyte antigen antibodies are specific for predicting transplant glomerulopathy and late graft failure after kidney transplantation. Transplantation. 2011; 91: 342-347.

12. Park WD, Grande JP, Ninova D, et al. Accommodation in ABO-incompatible kidney allografts, a novel mechanism of self-protection against antibody-mediated injury. American journal of transplantation: official journal of the American Society of Transplantation and the American Society of Transplant Surgeons. 2003; 3: 952-960.

13. Soulillou JP, Giral M and Brouard S. Operational tolerance in kidney transplantation-improved terminology may enable more precise investigation. Transplantation. 2013; 96: e36-e38.

14. Lee JR, Bang $\mathrm{H}$, Dadhania $\mathrm{D}$, et al. Independent risk factors for urinary tract infection and for subsequent bacteremia or acute cellular rejection: a single-center report of 1166 kidney allograft recipients. Transplantation. 2013; 96: 732-738.

\section{ORCID}

Rui F. Silva (iD) 0000-0003-4244-2459

Miguel Relvas (iD) 0000-0002-2947-1610

Ana Nunes (iD) 0000-0002-3483-4981

José Silvano (iD) 0000-0002-5266-2300

Ana Beco (iD) 0000-0002-0860-4648

Manuela Bustorff (iD 0000-0003-4632-9031

Susana Sampaio (iD) 0000-0001-8626-9235

\section{Correspondence to:}

Rui Fernando Oliveira da Silva, MD

Serviço de Nefrologia do Centro Hospitalar de São João

Alameda Prof. Hernâni Monteiro, 4200-319 Porto

E-mail: ruinefro@gmail.com 